\title{
SYMMETRIZATION OF DISTRIBUTIONS AND ITS APPLICATION. II: LIOUVILLE TYPE PROBLEM IN CONVOLUTION EQUATIONS
}

\author{
BY
}

\author{
KUANG-HO CHEN
}

\begin{abstract}
The symmetrization of distributions corresponding to a bounded $n-1$ dimensional $C^{\infty}$-submanifold of a $C^{\infty}$-manifold is constructed. This device reduces the consideration of distributions in $R^{n}$ to the one of distributions in $R^{1}$, i.e. the symmetrized distributions. Using the relation between the inverse Fourier transform of a symmetrized distribution and the one of the original (nonsymmetrized) distribution, we determine the rate of decay at infinity of solutions to a general convolution equation necessary to assure uniqueness. Using a result in the division problem for distributions, we achieve the following result: If $u \in C\left(R^{n}\right)$ is a solution of the convolution equation $S * u=f, f \in \mathscr{D}\left(R^{n}\right)$, with some suitable $S \in \mathcal{E}^{\prime}\left(R^{n}\right)$, then $u \in \mathscr{D}\left(R^{n}\right)$, provided $u$ decays sufficiently fast at infinity.
\end{abstract}

0 . Introduction. The purpose is to solve the Liouville type problem in the convolution equations

$$
S * u=f, \quad f \in \mathfrak{D}\left(R^{n}\right),
$$

for some class of $S \in \mathcal{G}^{\prime}\left(R^{n}\right)$, i.e. what kind of decay of the solutions $u \in C^{0}\left(R^{n}\right)$ at infinity gives $u \in \mathscr{D}\left(R^{n}\right)$ or gives unique solution of $(0.1)$. Here $\mathscr{D}\left(R^{n}\right)$ and $\mathscr{G}\left(R^{n}\right)$ are spaces of $C_{0}^{\infty}\left(R^{n}\right)$ and $C^{\infty}\left(R^{n}\right)$ functions with the L. Schwartz topology, respectively; $\mathscr{D}^{\prime}\left(R^{n}\right)$ and $\mathscr{S}^{\prime}\left(R^{n}\right)$ are the corresponding conjugate spaces (see [9], [11]-[13], [18], [23], [24] or [26]). About the existence of solutions of equation (0.1), we can see papers of Leon Ehrenpreis ([4]-[8]), Lars Hörmander ([15]-[18]), B. Malgrange [22], M. I. Višik and G. I. Ėskin ([28] and [29]), or Z. Zieleźny ([30] and [31]). The above two questions are solved in Theorems 3.2 and 4.4. As consequences from the corollaries of the two theorems, we have the corresponding results about the Liouville type problem for partial differential equations in [3], [14] and [20]. To solve the problem, we extend the idea in [3] of the symmetrization of distributions corresponding to an $n-1$ dimensional bounded $C^{\infty}$-manifold which has no boundary and is imbedded in $R^{n}$ to the one corresponding to a bounded $C^{\infty}$-submanifold of an $n-1$ dimensional $C^{\infty}$-manifold imbedded in $R^{n}$ (in $\$ 1$ ). We also derive the properties, which are parallel to those in [3], characterizing a symmetrized distribution and its inverse Fourier transform (see $\$ 2$ ).

Received by the editors February 23, 1971.

AMS 1970 subject classifications. Primary 28A30, 35C15, 35D10, 35E99, 35G05; Secondary 28A10, 28A20, 28A30, 30A08, 30A64, 30A86.

Key words and phrases. Symmetrization of distributions corresponding to a manifold in distribution (or function) sense, convolution equation, Liouville type problem, $C^{\infty} \cdot$ dif- $^{-}$ feomorphism, division problem. 
Essentially, the consideration of the Liouville type problem for $(0.1)$ with $f \neq$ 0 and with $S \in \mathcal{S}_{k}, k>0$ (i.e. Theorem 4.4) concerns the division problem which has been solved in different fields, expecially by Leon Ehrenpreis in [4]-[8] and by Lars Hörmander in [15], [16] and [17]. We make use of the results, in particular, in [7] and [18]. The author wants to express his thanks to both of them.

The author would like to express his sincere acknowledgement to Professor $\mathrm{H}$. Toda because of his continuous encouragement, Dr. R. P. Boas and Avner Friedman because of their helpful advice, and Dr. J. R. Foote, his chairman, because of his kind help and of the assistance given to the author.

1. Symmetrization of distribution corresponding to a smooth manifold. Denote by $\mathcal{K}$ the class of functions $f \in C^{\infty}\left(R^{n}\right)$ satisfying the following two conditions:

(i) The null set, $N(f)=\left\{\xi \in R^{n}: f(\xi)=0\right\}$, of $f$ is nonempty;

(ii) $\operatorname{grad} f(\xi) \neq 0$ for each $\xi \in N(f)$.

For such a function $f$, we have the Inverse Mapping Theorem recalled as follows:

Theorem 1.1. Suppose at $\xi_{0} \in N(f)$,

$$
\partial f(\xi) / \partial \xi_{j} \neq 0 \text { for some } j, 1 \leq j \leq n \text {. }
$$

Then there is a $C^{\infty}$-diffeomorphism $t=t(\xi)$, defined by $t_{j}=f(\xi)$ and $t^{\prime}=\xi^{\prime}$ with $t^{\prime}=\left(t_{1}, \cdots, t_{j-1}, t_{j+1}, \cdots, t_{n}\right)$, which maps a neigbborbood $U$ in $R^{n}$ of $\xi_{0}$ onto a ball $B_{t\left(\xi_{0}\right), \epsilon\left(\xi_{0}\right)}$ with $t\left(\xi_{0}\right)$ as the center and $\epsilon\left(\xi_{0}\right)$ as the radius. Moreover, its inverse mapping $\xi=\xi(t)$, defined by $\xi_{j}=\xi_{j}(t), \xi^{\prime}=t^{\prime}$, is also a $C^{\infty}$-diffeomorphism.

Hence $N(f)$ is a $C^{\infty}$-manifold and the volume element $d \xi$ in $R^{n}$ can be expressed as

$$
d \xi=\left(\left.\frac{\partial f(\xi)}{\partial \xi_{j}}\right|_{\xi=\xi(t)}\right)^{-1} d t=d S_{q}(t) d q \quad \text { on } B_{t\left(\xi_{0}\right), \epsilon\left(\xi_{0}\right)}
$$

where

$$
d S_{q}(t)=\left(\left.\frac{\partial f(\xi)}{\partial \xi_{j}}\right|_{\xi=\xi(t)}\right)^{-1} d t^{\prime} \quad \text { when } t_{j}=f(\xi)=q .
$$

In general, for any $\xi_{0} \in N(f)$, we have $d \xi=d S_{q} \cdot d q$, with $|q|<\epsilon\left(\xi_{0}\right)$, for all $\xi \epsilon$ $U_{\xi 0^{\circ}}$ We call $d S_{q}$ the surface element of $N(f-q)$. Here $N(f-q)=\left\{\xi \in R^{n}: f(\xi)=q\right\}$, $q \in R^{1}$.

Let $b=\left(b_{1}, \cdots, b_{n}\right), b_{i}>0$, be a positive finite vector. Denote by $G_{b}$ the rectangular set

$$
G_{b}=\left\{\xi \in R^{n}:\left|\xi_{i}\right| \leq b_{i}, \quad i=1, \cdots, n\right\}
$$

Then $N(f) \neq \varnothing$ means there is a sufficiently large vector $b>0$ such that $N(f) \cap$ $G_{b} \neq \varnothing$. By the Inverse Mapping Theorem, for fixed $b>0$, there is a neighborhood 
$U_{\xi}$ of $\xi$ for each $\xi \in N(f) \cap G_{b}$ on which $C^{\infty}$ local coordinates exist. Let $\mathcal{F}_{b}$ be the union of all such neighborhoods $U_{\xi}, \xi \in N(f) \cap G_{b}$;

$$
\mathcal{F}_{b}=\bigcup_{\xi \in N(f) \cap G_{b}} U_{\xi} .
$$

Define $N_{b}(f-q)=N(f-q) \cap \overline{\mathcal{F}}_{b}$, where $\overline{\mathcal{F}}_{b}$ denotes the closure of $\mathcal{F}_{b}$. Then using the arguments in the proof of Lemma 1.1 in [3], we have

Lemma 1.1. For each $f \in \mathcal{K}$ with sufficiently large nonnegative finite vector $b$, there is a number $\epsilon>0$ such that $N_{b}(f-q)$ is a $C^{\infty}$-manifold for eacb $q \in[-\epsilon, \epsilon]$.

Denote by $U_{b, \epsilon}$ the interior of $U_{|q|<\epsilon} N_{b}(f-q)$. If $\epsilon>0$ is small enough, by the Heine-Borel Theorem, there is a finite number of points, say $\xi_{0}^{i}$, in $\bigcup_{|q| \leq \epsilon} N_{b}(f-q) \backslash U_{b, \epsilon}$ and a neighborhood $U_{\xi_{0}^{i}}^{\delta}$ of each $\xi_{0}^{i}$ corresponding to the disk $B_{t\left(\xi_{0}^{i}\right), \delta}$ which satisfy Theorem 1.1 with some sufficiently small $\delta>0$, such that $\left\{U_{\xi_{0}^{i}}^{\delta}\right\}$ forms an open covering of $U_{|q| \leq \epsilon} N_{b}(f-q) \backslash U_{b_{i}, \epsilon}$ With this $\delta$, let

$$
\bigcup_{|q|<\epsilon / 3} N_{b}(f-q){\overline{\bigcup_{i} U_{\xi_{0}^{i}}^{\delta}}}_{\underbrace{i}}(\neq \varnothing)
$$

be denoted by $W_{b, \epsilon, \delta}$. Similarly, there is a finite number of points $\xi^{j}$ in $U_{b, \epsilon} \backslash \bigcup_{i} U_{\xi_{0}^{i}}^{\delta}$ and a set of neighborhoods $U_{\xi^{j}}$ of $\xi^{j}$ such that $\left\{U_{\xi^{j}}\right\}$ forms an open covering of $\overline{U_{b, \epsilon}} \backslash \bigcup_{i} U_{\xi_{0}^{i}}^{\delta}$. Hence, $\left\{U_{\xi_{0}^{i}}^{\delta}\right\} \cup\left\{U_{\xi^{j}}\right\}$ forms an open covering of $\overline{\mathcal{F}}_{b}$. Define

$$
V_{b, \epsilon}=\left\{\bigcup_{j} U_{\xi^{j}}\right\} \cap U_{b, \epsilon / 2}
$$

from this definition it follows that $\bar{N}_{b}(f-q)=N(f-q) \cap \overline{V_{b, \epsilon}}$ is a $C^{\infty}$-manifold for each $q \in[-\epsilon, \epsilon]$, and the three open sets $W_{b, \epsilon, \delta}, V_{b, \epsilon}, U_{b, \epsilon}$ satisfy $\overline{W_{b, \epsilon, \delta}} \subset V_{b, \epsilon}$; $\overline{V_{b, \epsilon}} \subset U_{b, \epsilon}$. Let $\chi_{s}, \chi_{l}$ be two functions in $\mathscr{D}\left(R^{n^{\prime}}\right)$ such that $0 \leq \chi_{s} \leq 1, \chi_{s}=1$ on $W_{b, \epsilon, \delta}$, Supp $\chi_{s} \subset V_{b, \epsilon}$, and such that $0 \leq \chi_{l} \leq 1, \chi_{l}=1$ on $\overline{V_{b, \epsilon}}$ and Supp $\chi_{l} \subset$ $U_{b, \epsilon^{\bullet}}$

Definition 1.1. For each $\phi \in L_{\mathrm{loc}}\left(R^{n}\right)$, set

$$
\begin{aligned}
& \phi^{\rho(b)}(q)=\left\{\begin{array}{l}
\int_{N_{b}(f-q)}\left(\chi_{s} \phi\right)(t) d S_{q}(t) \text { if }|q| \leq \epsilon, \\
0 \quad \text { if }|q|>\epsilon ;
\end{array}\right. \\
& \phi^{\sigma(b)}(\xi)=\left\{\begin{array}{l}
\chi_{l}(\xi) \phi^{\rho(b)}(f(\xi)) \text { if } \xi \in U_{b, \epsilon}, \\
0 \quad \text { if } \xi \notin U_{b, \epsilon} .
\end{array}\right.
\end{aligned}
$$


We call $\phi^{\sigma(b)}$ the symmetrization of $\phi$ corresponding to the manifold $\overline{N_{b}(f)}$ (in the function sense). For any distribution $T \in \mathfrak{D}^{\prime}\left(R^{n}\right)$ with Supp $T \subset \overline{V_{b, \epsilon}}$, we define the symmetrization $T^{\sigma(b)}$ of $T$ corresponding to the manifold $N_{b}(f)$ as follows:

$$
\left(T^{\sigma(b)}, \phi\right)=\left(T, \phi^{\sigma(b)}\right), \quad \phi \in \mathscr{D}\left(R^{n}\right) \text {. }
$$

The arguments in the proof of Lemma 1.1 in [3] are similarly applicable here to show that the above definitions are meaningful. Further, since $\chi_{l}=1$ on Supp $T$, the definition of $T^{\sigma(b)}$ is independent of the choice of $\chi_{i}$.

The symmetrization defined here corresponds to a bounded $C^{\infty}$-submanifold $\overline{N_{b}(f)}$ of the $C^{\infty}$-manifold $N(f)$. This idea is more general and practicable than the definitions of symmetrization in [3], in which the manifold $N(f)$ needed to be bounded.

Definition 1.2. Let the Dirac-measures $\left(D^{b} \delta\right)_{b}(f)$ on $\overline{N_{b}(f)}$ be defined as follows:

$$
\left(\left(D^{b} \delta\right)_{b}(f), \phi\right)=\left(D^{b} \delta, \phi^{\rho(b)}\right), \quad \phi \in \mathscr{D}\left(R^{n}\right),
$$

where $\delta$ is the Dirac-measure in $R^{1}$.

With the change in the corresponding notation in the proof of Theorem 1.1 in [3], we have

Theorem 1.2. Let $v$ be a distribution with support contained in $\overline{N_{b}(f)}$ with $f \epsilon$ K. Then its symmetrization $v^{\sigma(b)}$ corresponding to $N_{b}(f)$ is a linear combination of Dirac-measures on $N_{b}(f)$

$$
v^{\sigma(b)}=\sum_{0 \leq b \leq n} C_{b}\left(D^{b} \delta\right)_{b}(f) \text { with } C_{b}=\frac{(-1)^{b}}{b !}\left(v, \chi_{l} f^{b}\right) \text {. }
$$

2. The Fourier transform of symmetrized distributions.

Definition 2.1. Let $\mathcal{S}$ be a class of all the distributions $S \in \mathcal{E}^{\prime}\left(R^{n}\right)$ such that their Fourier transforms $\hat{S}$ belong to $\mathcal{K}$, thus satisfying the conditions (i) and (ii); let $\mathfrak{S}_{k}, 0 \leq k<n$, be a subclass of $\mathbb{S}$ consisting of all $S \in \mathbb{S}$ which satisfy the condition

(iii) At each point of $N(\hat{S})$ at least $k$ of the $n-1$ principal curvatures of the manifold $N(\hat{S})$ are different from zero.

Since $S \in \mathcal{E}^{\prime}\left(R^{n}\right)$ has compact support, $\hat{S}(\xi)$ can be extended into an entire function $\hat{S}(\xi+i \eta)$, and then the above definition is meaningful. From the definitions of $\left(\pi_{k}\right)$ in [3], the main difference between $\left(\mathcal{E}_{k}\right.$ and $\left(\pi_{k}\right)$ is the requirement that $N(P)$ is bounded if $P \in\left(\pi_{k}\right)$. Incidentally, $\left(\pi_{k}\right)$ is a proper subclass of $\mathcal{E}_{k}$ for each $k$ and there is at least a polynomial in $\mathcal{F}_{k}$ for each $n>k \geq 0$.

With arguments similar to those in the proof of Lemma 2.2 in [3], we have

Lemma 2.1. For every $S \in \mathfrak{S}_{k}, n>k \geq 0$, and for each sufficiently large positive vector $b$, there is an $\epsilon>0$ sucb that for eacb $|q|<\epsilon, \overline{N_{b}}(\hat{S}-q)$ satisfies the condition (iii). 
Let the constant $\epsilon>0$ satisfy the assertions in Lemmas 1.1 and 2.1 and let $E_{b}(x, \xi)=\chi_{s}(\xi) e^{i x \cdot \xi}$. Then for each $x \in R^{n}$, we have defined $E_{b}^{\rho(b)}(x, \cdot)$ and the symmetrization $E_{b}^{\sigma(b)}(x,$.$) of E_{b}(x,$.$) corresponding to the manifold \overline{N_{b}}(\hat{S})$.

For a given unit vector $\omega \in R^{n}$ and fixed number $q,|q|<\epsilon$, there are finite number of points $p_{j}(q, \omega)$ on $\overline{N_{b}}(\hat{S}-q)$ at which the normal of $N_{b}(\hat{S}-q)$ is $\omega$ provided $k=n-1$. In case $0<k<n-1$, the situation is no more true. There is a compact set $A$ of points on $N_{b}(\hat{S}-q)$ satisfying the property if $\omega$ is in the direction of the normal of $\bar{N}_{b}(\hat{S}-q)$ in $A$. By the Heine-Borel Theorem, there are finite number of points $p_{j}$ with small neighborhoods $U_{j}$ which form a covering of $A$. By partition of unity, there are $\phi_{j} \in C_{0}^{\infty}\left(R^{n}\right), 0 \leq \phi_{j} \leq 1$, supp $\phi_{j} \subset U_{j}$ and $\Sigma \phi_{j}=1$ on $A$. Let us denote by $d_{+}\left(p_{j}(q, \omega)\right)$ and $d_{-}((q, \omega))$ the number of the positive and the negative corresponding principal curvatures, respectively. We want to remark that, in case $0<k<n-1, U_{j}$ can be chosen such that $d_{+}\left(p_{j}(q, \omega)\right)$ and $d_{-}\left(p_{j}(q, \omega)\right)$ depend only on $U_{j}$ and are independent of the choice of $p_{j}(q, \omega) \in U_{j}$. The details appear in the appendix.

Theorem 2. 1. With sufficiently small number $\epsilon>0$, for eacb $q,|q|<\epsilon$, and unit vector $\omega \in R^{n}$ in the direction of the outward normal of $N(\hat{S})$ at points in $N(\hat{S})$ $\cap$ Supp $X_{s}$, where the number of nonzero principal curvatures is $k$,

$$
\begin{aligned}
& E^{\rho(b)}(|x| \omega, q) \\
&=\left(\frac{\pi}{2}|x|^{-1}\right)^{k / 2} \\
& \times \sum_{j}(1+\tau)^{\left.d_{+}(p, j, \omega)\right)}(1-\tau)^{d_{-}\left(p_{j}(q, \omega)\right)} C_{s, j}(q, \omega) \exp \left\{i p_{j}(q, \omega) \cdot \omega|x|\right\} \\
&+O\left(|x|^{-(k+1) / 2}\right)
\end{aligned}
$$

with some $C_{s, j}(q, \omega)$, which is $\left|K\left(p_{j}(q, \omega)\right)\right|^{-1 / 2}$ when $k=n-1$, where the summation is a finite sum and $K(\xi)$ is the Gaussian curvature of $N(\hat{S})$ at $\xi$.

Lemma 2.2 Let $S \in \mathcal{S}_{k}$ with the largest $k$ and let $W_{b}(x, y)$ be the inverse Fourier transform $\mathcal{F}^{-1}\left\{E_{b}^{\sigma}(x),\right\}(y)$ of $E^{\sigma(b)}(x$,$) . Then for a sufficiently small$ number $\epsilon>0$ satisfying the assertions in Lemmas 1.1 and 2.1,

$$
W_{b}(x, y)=\int_{-\epsilon}^{\epsilon} E_{b}^{\rho}(x, q) E_{b}^{\rho}(y, q) d q
$$

and, for any integer $\rho \geq 0$,

$$
W_{b}\left(|x| \omega,|y| \omega^{\prime}\right)=O\left(|x|^{-p-k / 2}|y|^{p-k / 2}[|x|+|y|]^{-1}\right)
$$

when $\left(|x| \omega,|y| \omega^{\prime}\right) \rightarrow \infty$ in the direction $\left(\omega, \omega^{\prime}\right)$. Here $\omega, \omega^{\prime}$ are any pair of unit vectors in $R^{n}$ if $k=n-1$ and are a pair of unit vectors in $\Omega$, which is $\Omega(q)$ in Theorem 1.2 which is independent of $q$ with $|q|<\epsilon$ if $k<n-1$. 
With arguments similar to those in the proofs of Lemma 2.1 and Theorem 2.1 in [3], we have the result. Using arguments similar to those used in the proofs of Theorems 3.1 and 4.1 in [3], we have the following two theorems.

Theorem 2.2 With $S \in \mathbb{S}_{k}, k>0$, let $v$ be the Fourier transform of a distribution $u \in \mathfrak{D}^{\prime}\left(R^{n}\right)$ with support contained in $W_{b, \epsilon, \delta}$. Then the inverse Fourier transform $u_{b}$ of the symmetrization $v^{\sigma(b)}$ corresponding to $N_{b}(\hat{S})$ can be expressed in the form

$$
u_{b}(x)=\int_{R^{n}} u(y) W_{b}(y, x) d y .
$$

Here $W_{b}(y, x)$ is as in Lemma 2.2 and $W_{b, \epsilon, \delta}$ is constructed in Definition 1.1 with $f=\hat{S}$ with small $\epsilon>0$ and $\delta>0$.

Theorem 2.3. Let the support of the Fourier transform $v$ of a.distribution $u \epsilon$ $\mathscr{D}\left(R^{n}\right)$ be contained in $N_{b}(\hat{S}), S \in \mathbb{S}_{k}$ with largest $k(0<k<n)$ and let $u_{b}$ be the inverse Fourier transform of the symmetrization $v^{\sigma(b)}$ of v corresponding to $N_{b}(\hat{S})$. Then when $x \rightarrow \infty$,

$$
\begin{aligned}
& u_{b}(x)=O\left(|x|^{-d}\right) \text { uniformly in direction, } \\
& u_{b}(|x| \omega) \neq O\left(|x|^{-d}\right) \text { for some unit vectors } \omega \text {, if } k=n-1 \text {, }
\end{aligned}
$$

for some $d \leq k / 2$, provided $u_{b} \neq 0$; on the other band, when $x \rightarrow \infty$,

$$
u_{b}(x)=o\left(|x|^{n-1-k-b}\right) \quad \text { if } u(x)=o\left(|x|^{-b}\right), b \geq 0 .
$$

Lemma 2.3. Under the conditions of Theorem 1.3, we bave $u(0)=u_{b}(0)$.

Indeed, its proof is in the proof of Lemma 6.1 in [3].

Remark. Since the arguments in the proofs of Theorems 2.2 and 2.3 are not applicable for the case $k=0$, especially in the concerned integrations, we exclude the case in the statements. On the other hand, there are some special geometric meanings for this case. We will discuss it in the next paper.

3. An application of the symmetrization. Since the convolution of distributions possesses a very nice property (3.2) about translation in coordinates, we are discussing the translation invariance of solutions to convolution equations

$$
S * u=0 \text {. }
$$

With $\eta \in R^{n}$ and any function $u$, we denote by $u_{\eta}$ the translation of $u$ by $\eta$ defined as $u_{\eta}(\xi)=u(\xi-\eta)$.

Definition 3.1. Let $T \in \mathscr{D}^{\prime}\left(R^{n}\right)$ be a distribution. Define its translation $T_{\eta}$ by $\eta \in R^{n}$ as follows: $\left(T_{\eta}, \phi\right)=\left(T, \phi_{\eta}\right), \phi \in \mathscr{D}\left(R^{n}\right)$.

Then if $u * v$ is well defined for two distributions $u$ and $v$ in $\mathfrak{D}^{\prime}\left(R^{n}\right)$ we have

$$
u_{\eta} * v=(u * v)_{\eta}=u * v_{\eta}
$$


Lemma 3.1. If $S \in \mathcal{E}^{\prime}\left(R^{n}\right)$ and if $u \in \mathscr{D}^{\prime}\left(R^{n}\right)$ is a solution of the convolution equation (3.1), then its translation $u_{\eta}$ with any $\eta \in R^{n}$ is a solution of the equation (3.1).

Proof. It is well known [9] that $T * \delta=T=\delta * T, T \in \mathfrak{D}^{\prime}\left(R^{n}\right)$. Then by compactness of Supp $\delta_{\eta}$ and Supp $S$, (3.2) yields

$$
S * u_{\eta}=[\delta * S]_{*} u_{\eta}=\delta_{\eta} *[S * u]=\delta_{\eta} * 0=0, \quad \eta \in R^{n}
$$

Hence for each $\eta \in R^{n}, u_{\eta}$ is a solution of (3.1) if $u$ is. This completes the proof.

By this lemma, we want to discuss a uniqueness property of homogeneous convolution equations (2.1).

Lemma 3.2. Let $S \in \mathbb{E}_{n-1}$ be a distribution and $u$ be a solution of the convolution equation (2.1). Then $u=0$ if $u(x)=o\left(|x|^{-d}\right)$ with $d \geq(n-1) / 2$ when $x \rightarrow \infty$.

Before proving the lemma, we recall some properties from Avner Friedman [9] about convolutions which are needed later.

Definition 3.2 Let $\Phi$ denote the topological space either $\mathcal{S}\left(R^{n}\right)$ or $\mathscr{D}\left(R^{n}\right)$ and $\Phi^{\prime}$ denote its conjugate space. We say that a distribution $u \in \Phi^{\prime}$ convolves with $\Phi$ if, for any $\phi \in \Phi, u * \phi(x)=u * \phi_{-x}=(u(y), \phi(y+x))$ belongs to $\Phi$ and the mapping $\phi \rightarrow u * \phi$ is a continuous mapping from $\Phi$ into $\Phi$.

Theorem 3.1. Let $g(x), b(x)$ be functionals in $\mathcal{S}^{\prime}\left(R^{n}\right)$ of function type and suppose that $g$ convolves with $\mathcal{S}\left(R^{n}\right)$ and that the classical convolution $g \circledast b$ exists as a function $k(x)$ in $\mathcal{S}^{\prime}\left(R^{n}\right)$. If

$$
\int_{R^{n}} \int_{R^{n}}|b(x) g(y-x) \phi(y)| d x d y<+\infty
$$

for all $\phi \in S\left(R^{n}\right)$, then the convolution $g * b$ in the distribution sense is just $g \circledast b$.

Proof of Lemma 3.2 Taking the Fourier transform on both sides of the convolution equation (3.1), we have $\hat{S} \cdot \hat{u}=0$. Then Supp $\hat{u} \subset N(\hat{S})$. For any sufficiently large finite nonnegative vector $b$, let $\hat{\kappa}_{b} \in \mathscr{D}\left(R^{n}\right)$ be a function such that $1 \geq \hat{\kappa}_{b} \geq 0$, Supp $\hat{\kappa}_{b} \subset W_{b, \epsilon, \delta}$ which is constructed in $\$ 1$. Hence

$$
\hat{S} \cdot\left(\hat{u} \hat{\kappa}_{b}\right)=0 .
$$

Let $v_{b}$ and $\kappa_{b}$ be the inverse Fourier transforms of $\hat{u} \hat{\kappa}_{b}$ and $\hat{\kappa}_{b}$, respectively. Therefore $v_{b}=u * \kappa_{b}$, and by (3.3), $v_{b}$ is a solution of the convolution of (3.1). Theorem 3.1 implies $u * \kappa_{b}=u \circledast \kappa_{b}$. Then by $\kappa_{b} \in S\left(R^{n}\right)$, when $x \rightarrow \infty, v_{b}(x)=$ $o\left(|x|^{-d}\right)$. Let $u^{\sigma(b)}$ be the symmetrization of $\hat{v}_{b}=\hat{u} \hat{\kappa}_{b}$ corresponding to the manifold $N_{b}(\hat{S})$ and $u_{b}$ be its inverse Fourier transform $\mathfrak{F}^{-1}\left\{\hat{u}^{\sigma(b)}\right\}$. Hence Theorem 2.3 implies $u_{b}(x)=o\left(|x|^{n-1-k-d}\right)$ when $x \rightarrow \infty$; on the other hand, if $u_{b} \neq 0$, $u_{b}(x)=O\left(|x|^{-b}\right)$ and $u_{b}(x) \neq o\left(|x|^{-b}\right), b \leq(n-1) / 2$, when $x \rightarrow \infty$. Since $d \geq$ $(n-1) / 2, n-1-k-d \leq-(n-1) / 2 \leq-b$. Hence $u_{b}=0$. Lemma 2.3 yields 
$v_{b}(0)=u_{b}(0)$ and then $v_{b}(0)=0$. Therefore this and Lemma 3.1 imply $v_{b}(x)=0$ for any $x \in R^{n}$ and any large $b$. Thus $u=0$. This completes the proof.

All the previous discussion from $\$ 1$ is a kind of preparation to prove the consequences we will get here, although each of the preceding results has its own interest. We discuss now the uniqueness problem.

We consider the distribution $S \in \mathcal{E}^{\prime}\left(R^{n}\right)$ satisfying the following two conditions:

(I) There is a factor $\hat{\Theta}$ of $\hat{S}$ such that $N(\hat{\Theta}) \neq \varnothing$.

(II) For each irreducible factor $\Theta$ of $\hat{S}$ such that $N(\hat{\Theta}) \neq \varnothing, \Theta \in \mathbb{S}_{n-1}$.

Theorem 3.2. The function $u=0$ is the only solution, in the distribution sense, of (3.1) such that $u \in C\left(R^{n}\right)$ and $u(x)=o\left(|x|^{-d}\right), d \geq(n-1) / 2$, when $x \rightarrow \infty$.

Proof. Since $S \in \mathcal{E}^{\prime}\left(R^{n}\right)$ means Supp $S$ compact, its Fourier transform $\hat{S}$ is an entire function of finite exponential type. Then we can write $\hat{S}$ in the form $\hat{S}=$ $\hat{S}_{0} \Pi_{1<_{j} \leq_{r}}\left[\hat{S}_{j}\right]^{r j}, r \geq 1$; and therefore

$$
S=S_{0} * \prod_{1 \leq j \leq r}^{*}\left[S_{j}\right]_{*}^{r}
$$

where

$$
\begin{array}{r}
\quad\left[S_{j}\right]_{*}^{r} \equiv S_{j} * \cdots * S_{j} \text { with } r_{j} \text { factors, } \\
\prod_{1 \leq j \leq r}^{*}\left[S_{j}\right]_{*}^{r} \equiv\left[S_{1}\right]^{r}{ }^{r} * \cdots *\left[S_{r}\right]^{r} ;
\end{array}
$$

$S_{j} \in \mathcal{C}_{k_{j}}, 0<k_{j}<n, j=1, \ldots, r$, with $N\left(\hat{S}_{j}\right)$ distinct from each other; $k=$ $\min _{1 \leq j \leq r} k_{j}$; and $S_{0} \in \mathcal{G}^{\prime}\left(R^{n}\right)$ with $N\left(\hat{S}_{0}\right)=\varnothing$. Taking the Fourier transform on both sides of the equation (3.1), we have $\hat{S} \cdot \hat{u}=0$. Hence Supp $\hat{u} \subset N(\hat{S})$. Assume $u \neq 0$, then Supp $\hat{u} \neq \varnothing$. There is a sufficiently large finite nonnegative vector $b=\left(b_{1}\right.$, $\left.\cdots, b_{n}\right)$ such that Supp $\hat{u} \cap G_{b} \neq \varnothing$. Let the function $\kappa_{b} \in \mathcal{D}\left(R^{n}\right)$ be with support in $G_{b+\epsilon}, \epsilon>0,1 \geq \hat{\kappa}_{b} \geq 0$ and $\hat{\kappa}_{b}=1$ on $G_{b}$. Then with $\hat{\kappa}_{b} \in \mathcal{S}\left(R^{n}\right)$ as the inverse Fourier transform of $\hat{\kappa}_{b}$, we have $\hat{S} \cdot \hat{u} \hat{\kappa}_{b}=0$ and $S * v_{b}=0$ with $v_{b}=u * \kappa_{b}$. Since $v_{b}=$

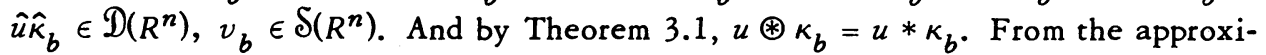
mation in the integral expression of $u * \kappa_{b}$, by $u \in \mathcal{C}\left(R^{n}\right)$, we see that $v_{b}(x)=$ $o\left(|x|^{-d}\right)$ when $x \rightarrow \infty$.

(Remark. If $N(S)$ is bounded, i.e. $N\left(S_{j}\right)$ are bounded, $j=1, \cdots, r$, then we choose the $b$ so large that $N(S) \subset G_{b}$. Therefore we can see that $v_{b}=u$, and the argument up to now is not necessary.)

On the other hand,

$$
\begin{aligned}
S * v_{b} & =\mathcal{F}^{-1}\left\{\hat{S} \cdot\left(\hat{u} \hat{\kappa}_{b}\right)\right\} \\
& =\mathcal{F}^{-1}\left\{\hat{S}_{r} \cdot\left(\hat{S}_{r}^{\prime} \hat{\kappa}_{b}\right) \cdot \hat{u}\right\}=S_{r} * f
\end{aligned}
$$

with $\hat{S}_{r}^{\prime}=\hat{S} / \hat{S}_{r}$ (it makes sense, since $\hat{S}^{1}$ and $S_{r}$ are entire functions) and $f=$ $\mathcal{F}-1\left\{\left(\hat{S}_{r}^{\prime} \hat{\kappa}_{b}\right) \cdot \hat{u}\right\}$. Then since the Fourier transform $\hat{f}$ of $f$ has compact support and 
is in $\mathcal{E}^{\prime}\left(R^{n}\right), f(x)$ is an analytic function; moreover, $f(x)$ is a solution of $S_{r} * f=0$, $S_{r} \in \mathbb{S}_{n-1}$. However, $\hat{f}=\left(\hat{S}_{r}^{\prime} \hat{\kappa}_{b}\right) \cdot \hat{u}$. Since the inverse Fourier transform $\hat{S}_{r}^{\prime}$ of $S_{r}^{\prime} \epsilon$ $\tilde{E}^{\prime}\left(R^{n}\right)$ is analytic on $R_{n}, \hat{S}_{r}^{\prime} \hat{\kappa}_{b}$ belongs to $\mathcal{D}\left(R^{n}\right)$. Hence with the same arguments for $v_{b}, f(x)=\mathcal{F}-1\left\{\hat{S}_{r}^{\prime} \hat{\kappa}_{b}\right\} * u(x)$ and $f(x)=o\left(|x|^{-d}\right)$ when $x \rightarrow \infty$. Further, $d \geq n-$ $1-(n-1) / 2 \geq(n-1) / 2$. Lemma 3.2 yields $f=0$.

Eventually we arrive at

$$
S_{r}^{\prime} * v_{b}=\mathcal{F}^{-1}\left\{\hat{S}_{r}^{\prime} \cdot\left(\hat{\kappa}_{b} \hat{u}\right)\right\}=\mathfrak{F}^{-1}\left\{\left(\hat{S}_{r}^{\prime} \hat{\kappa}_{b}\right) \cdot \hat{u}\right\}=f=0 .
$$

Repeating the above arguments, $\Sigma_{1 \leq j \leq r} r_{j}$ times, we conclude $S_{0} * v_{b}=0$. But by taking the Fourier tranform on both sides, Supp $\check{v}_{b} \subset N\left(\check{S}_{0}\right)=\varnothing$; that is, $\operatorname{Supp}\left(\hat{u} \hat{\kappa}_{b}\right)=\varnothing$ for any finite nonnegative vector $b$. This contradicts Supp $\hat{u} \neq \varnothing$. Hence $u=0$.

Remark. If we let $S=P(D) \delta, D_{\boldsymbol{x}}=-i \partial / \partial x$, as an application, with differential polynomial $P(D)$, we see that the conclusions of Theorem 3.2 are just for partial differential equations. And all the corresponding results in Walter Littman [20] and $\mathrm{K}$. Chen [3] follow from them.

4. Liouville type problems. We want to consider the problem: What kind of decay at infinity for a solution $u$ of the convolution equation

$$
S * u=f, \quad f \in \mathcal{D}\left(R^{n}\right),
$$

gives $u \in \mathscr{D}\left(R^{n}\right)$ for some $S \in \mathscr{D}^{\prime}\left(R^{n}\right)$ ? This shall be answered by a criterion appearing later, which, as we mentioned in the Introduction, essentially reduces to a form of the division problem (see [4] to [8] by Leon Ehrenpreis, [16] and [17] by Lars Hörmander).

For the need in the proof of the criterion, we give some preliminary preparation here. Using the usual definition (see [2]) of an irreducible function with $n$ variables, we see that the proof of a result in F. Tréves [25, p. 107] (or [3]) gives the following extended

Theorem 4.1. Let $G$ be an irreducible entire function in the class $K$ and $V$ be the set of zeros of $G(z)$ in $C^{n}$. Let $F$ be an entire function on $C^{n}$.

Assume that the function $F / G$ defined in $C^{n}-V$ can be extended, as a bolomorphic function, to an open set $\Omega$ intersecting $V$; then $F / G$ can be extended to $C^{n}$ as an entire function.

We recall, further, some idea from Leon Ehrenpreis [7] (see also [17]) as follows:

Definition 4.1. Let $S \in \mathcal{G}^{\prime}\left(R^{n}\right)$; then the function $\hat{S}$ is called slowly decreasing if there exists a positive number $a$ such that for each point $\xi \in R^{n}$ we can find a point $\eta \in R^{n}$ satisfying $|\xi-\eta| \leq a \log (1+|\xi|)$ and $|\hat{S}(\eta)| \geq(a+|\eta|)^{-a}$. The distribution $S$ is then called invertible.

Theorem 4.2 (Leon Ehrenpreis [7]). The following two properties for $S \epsilon$ $\mathcal{E}^{\prime}\left(R^{n}\right)$ are equivalent: 
(a) $S$ is invertible.

(b) For any entire function $G$, if the inverse Fourier transform $\mathcal{F}-1\{\hat{S} G\}$ of $\hat{S} G$ is in $\mathscr{D}\left(R^{n}\right)$, then $\mathcal{F}^{-1}\{G\} \in \mathcal{D}\left(R^{n}\right)$.

Let the distribution $S \in \mathcal{E}^{\prime}\left(R^{n}\right)$ satisfy the condition (I) and let the inverse Fourier transform $\Theta$ of every irreducible factor $\hat{\Theta}$ of its Fourier transform $\hat{S}$, such that $N(\hat{\Theta}) \neq \varnothing$, be in $\mathcal{S}$. Therefore it can be written in the form of (3.4), i.e.

$$
S=S_{0} * \prod_{1 \leq j \leq r}^{*}\left[S_{j}\right]_{*}^{r}
$$

with some integers $r \geq 1, r_{j} \geq 1, j=1, \cdots, r$, where $S_{j} \in \mathcal{S}$ and invertible with the $N\left(\hat{S}_{j}\right)$ distinct from each other.

For each $\xi_{0} \in N(\hat{S})$, there are integers $\mu_{0}, 1 \leq \mu_{0} \leq r$ and $1 \leq i_{1}<\ldots<i_{\mu_{0}} \leq r$, such that

$$
\xi_{0} \in N_{i_{1} \ldots i_{\mu_{0}}} \equiv \bigcap_{1 \leq i \leq \mu_{0}} N\left(S_{i_{j}}\right)
$$

and $\xi_{0} \notin N\left(\hat{S}_{i}\right)$ if $i \neq i_{1}, \cdots, i_{\mu_{0}}$. Since $S_{i} \in \mathcal{S}$, by Theorem 1.1 (i.e. the Implicit Mapping Theorem), there is a neighborhood $U_{\xi_{0}, i_{j}}$ of $\xi_{0}$ such that $\partial \hat{S}_{i_{j}}(\xi) / \partial \xi_{\nu} \neq 0$ on $U_{\xi 0, i j}$, with some integer $\nu=\nu\left(i_{j}\right), 1 \leq \nu \leq n$, and there is a $C^{\infty}$-diffeomorphism $t=t(\xi): t_{\nu}=\hat{S}_{i_{j}}(\xi), t_{i}=\xi_{i}, i \neq \nu$ such that $t(\xi)$ maps $U_{\xi_{0, i}}$ onto a neighborhood of $t\left(\xi_{0}\right)$ and whose inverse mapping is $\xi=\xi(t): \xi_{\nu}(t) \in C^{\infty}$ locally and $\xi^{\prime}=$ $t^{\prime}$. For any $\phi \in C^{\infty}\left(R^{n}\right)$, we can define on $U_{\xi_{0, i}}$

$$
\left.D_{i_{j}}^{b} \phi(\xi) \equiv D_{t_{\nu}}^{b} \phi\left(t_{1}, \cdots, t_{\nu-1}, \xi_{\nu}^{\cdot}(t), t_{\nu+1}, \cdots, t_{n}\right)\right|_{t=t(\xi)}
$$

Then on $U_{\xi_{0}}=\bigcap_{1 \leq j \leq \mu_{0}} U_{\xi_{0, i} ;}$,

$$
D_{i}^{b_{1}} \ldots D_{i_{\mu}}^{b_{\mu_{0}}} \phi(\xi)
$$

is meaningful. Now we mention the criterion below.

Theorem 4.3. Let the inverse Fourier transform $S_{j}$ of each irreducible factor $\hat{S}_{j}, j=0, \cdots, r$, of $\hat{S}$ such that $\dot{N}\left(\hat{S}_{j}\right) \neq \varnothing$ be invertible. The necessary and sufficient conditions for the existence of a solution $u$ in $\mathcal{D}\left(R^{n}\right)$ to the convolution equation (4.1) are the following:

For each $\xi_{0} \in N(\hat{S})$, with the corresponding integers $\mu: 1 \leq \mu \leq r, i_{j}: 1 \leq i_{1}<$ $\cdots<i_{\mu} \leq r$ and neighborbood $U_{\xi_{0}}$, on $U_{\xi_{0}} \cap N_{i_{1}} \cdots{ }_{i \mu}$, 


$$
D_{i}^{b}{ }_{\mu}^{b} \ldots D_{i}^{b}[\mathfrak{F}\{f\}](\xi)=0
$$

with $0 \leq b_{\mu}<s_{\mu}, 0 \leq b_{j} \leq s_{j}, 1 \leq j<\mu$.

Proof. The arguments for the necessity are the same as the corresponding part in the proof of the criterion in [3]. Next, we assume the conditions. Consider the equation (4.1) with $S=S_{1}$. Taking the Fourier transform, we have $\hat{S}_{1} \cdot \hat{u}=\hat{f}, f \epsilon$ $\mathcal{D}\left(R^{n}\right)$. By the arguments in [3] with the Tréves' Theorem replaced by Theorem 4.1, we see that the function $\hat{f}_{1}(z) \equiv \hat{f}(z) / \hat{S}_{1}(z)$ is an entire function on $C^{n}$. Since then $\mathcal{F}-1\left\{\hat{f}_{1} \hat{S}_{1}\right\} \equiv \mathcal{F}-1\{\hat{f}\}=f \in \mathfrak{D}\left(R^{n}\right)$ and $S_{1}$ is invertible, Theorem 4.2 yields $f_{1}=$ $\mathcal{F}-1\left\{\hat{f}_{1}\right\} \in \mathscr{D}\left(R^{n}\right)$. On the other hand, $S_{1} * f_{1}=f$. It is seen that $f_{1}$ satisfies the conditions with $r_{1}$ replaced by $r_{1}-1$. Repeating the above argument, just similar to the proof in [3], we have some $u \in \mathscr{D}\left(R^{n}\right)$ which is a solution of the equation (4.1). This completes the proof.

As an application of the criterion, we answer the question raised at the beginning of this section.

Theorem 4.4. Let $S$ be the distribution in Theorem 4.3. If $u \in \mathcal{C}\left(R^{n}\right)$ is a solution of the convolution equation (4.1) such that when $x \rightarrow \infty$

$$
u(x)=o\left(|x|^{-d}\right), \quad d \geq(n-1) / 2,
$$

then $u \in \mathscr{D}\left(R^{n}\right)$.

Corollary. With $S=P(D) * \delta, \delta$ being the Dirac-measure in $R^{n}$, the solution $u$ of the partial differential equation $P(D) u=f, f \in \mathcal{D}\left(R^{n}\right)$, is in $\mathscr{D}\left(R^{n}\right)$ if $u \in \mathcal{C}\left(R^{n}\right)$ and satisfies the condition (4.3) when $x \rightarrow \infty$.

Indeed, the corollary follows from the theorem directly since it is easily checked that a polynomial is slowly decreasing. With Theorem 3.2 and the criterion replacing the corresponding theorems in the proof of Theorem 6.1 in [3] we have the proof of the theorem.

Remark. The corresponding assertions in [3] or in [20] are subsequences of the corollary.

Before the end of this paper, we want to recall

Theorem 4.5 (Lars Hörmander [17]). Assume that $S \in \mathcal{G}^{\prime}\left(R^{n}\right)$ is not invertible. To every compact set $K$ in $R^{n}$ with interior points one can then find a continuous function $\phi$ with support in $K$ such that $S * \phi \in C_{0}^{\infty}\left(R_{n}\right)$ but $\phi$ is not in $C^{1}$.

This implies that the assumption, i.e. the inverse Fourier transform $S_{j}$ of each irreducible factor $\hat{S}_{j}$ of $\hat{S}$ is invertible, of Theorem 4.4 is sufficient. For $f=S * \phi$ $\in \mathscr{D}\left(R^{n}\right)$. But Theorem 4.4 yields $\phi \in \mathscr{D}\left(R^{n}\right)$. This contradicts $\phi \notin C^{1}$. 
5. Appendix. For $S \in \mathfrak{F}_{k}$, let $\mu$ be a smooth density function with bounded support on $N(\tilde{S})$ such that the set $N_{k}$ of points $p$ on $N(\widetilde{S}) \cap$ supp $\mu$, at which there are exactly $k$ nonzero principal curvatures of $N(\tilde{S})$, is not empty. Denote by $\Omega_{k}$ the unit (outward) normals $\Re(p)$ of $N(\tilde{S})$ at $p \in N_{k}$.

For a given unit vector $\omega$ which is not orthogonal to all vectors in $\Omega_{k}$, say $\Re(p)$ for some $p \in N_{k}$ (under the rotation we can assume that $\omega=(0, \cdots, 0,1)$ ), there is a neighborhood $U$ of $p$ of which each point can be expressed by

$$
\xi=\left(\xi^{\prime}, \xi_{n}\left(\xi^{\prime}, 0\right)\right) \text { with } \xi^{\prime}=\left(\xi_{1}, \ldots, \xi_{n-1}\right),
$$

where $\xi_{n}\left(\xi^{\prime}, 0\right)$ is an analytic function of $\xi^{\prime}$. By Taylor's formula, we have

$$
\xi_{n}\left(\xi^{\prime}, 0\right)=\xi_{n}(p)+\operatorname{grad}_{\xi^{\prime}} \xi_{n}(p) \cdot \xi^{\prime}+\sum_{a_{i j}} \xi_{i} \xi_{j}+O\left(|\xi|^{3}\right) .
$$

Since $0 \equiv \tilde{S}\left(\xi^{\prime}, \xi_{n}\left(\xi^{\prime}, 0\right)\right)$, for $j=1, \cdots, n-1, \partial \xi_{n}(p) / \partial \xi_{j}=-\tilde{S}_{j}(p) / \tilde{S}_{n}(p)$, where $\tilde{s}_{l}(\xi)=\partial \tilde{S}(\xi) / \partial \xi_{l}, l=1, \ldots, n$,

$$
\xi_{n}\left(\xi^{\prime}, 0\right)=\xi_{n}(p)-\frac{1}{S_{n}(p)} \sum_{j=1}^{n-1} \tilde{S}_{j}(p) \xi_{j}+\sum a_{i j} \xi_{i} \xi_{j}+O\left(|\xi|^{3}\right)
$$

Denoted by

$$
f\left(\xi^{\prime}\right)=\xi_{n}\left(\xi^{\prime}, 0\right)-\xi_{n}(p)+\frac{1}{\tilde{s}_{n}(p)} \sum_{j=1}^{n-1} \tilde{s}_{j}(p) \xi_{j}
$$

Then by a Morse Lemma, with $s_{j}$ at the direction of the $j$ th nonzero principal curvature of $N(\tilde{S})$ at $p$ and suitable choice of coordinates $\eta=(s, t), s=\left(s_{1}, \cdots, s_{k}\right)$, $t=\left(\eta_{k+1}, \cdots, \eta_{n-1}\right)$,

$$
f\left(\xi^{\prime}(n)\right)=\sum_{j=1}^{k} \lambda_{j}(t) s_{j}^{2}
$$

and the Jacobian $J(\xi, \eta)$ of the transform $\xi \rightarrow \eta$ is 1 at $p$. On a sufficiently small neighborhood, say $U$ of $p,|J(\xi, \eta)|>1 / 2$. Let $\phi \in C_{0}^{\infty}(U)$ be with support in $\{\xi=\xi(s, t) \in U,|t|<\epsilon\}$ for some number $\epsilon>0$. Since principal curvatures are continuous, we can assume that on $U$ the nonzero principal curvatures do not vanish and do not change sign. Then we set $d^{ \pm}$to be the numbers of positive and negative principal curvatures of $N(\tilde{S})$ on $U$, respectively, and let $K_{k}(\eta)$ be the product of the $k$ principal curvatures. Then we have the following approximation.

Lemma 5. 1. For $x=|x| \omega$ approaching infinity, 


$$
\begin{aligned}
& \int_{N(\widetilde{S})} e^{i x \cdot \xi} \mu(\xi) \phi(\xi) d S(\xi) \\
& =\left(\frac{\pi}{2}|x|^{-1}\right)^{k / 2}(i+i)^{d^{+}}(1-i)^{d^{-}} \exp \left\{i|x| \xi_{n}(p)\right\} \\
& \times \int_{|t| \leq \epsilon} \mu_{1}(t) \exp \left\{\frac{-i|x|}{\tilde{S}_{n}(p)} \sum_{j=1}^{n-1} \tilde{s}_{j}(p) \xi_{j}(0, t)\right\} d t \\
& +O\left(|x|^{-(k+1) / 2}\right)
\end{aligned}
$$

where $d S$ is the area element on the manifold $N(\tilde{S})$ and

$$
\mu_{1}(t)=\left.\mu(\xi) \phi(\xi) J(\xi, \eta)\right|_{\xi \doteq(0, t)}\left|K_{k}(0, t)\right|^{1 / 2} .
$$

Proof. By the assumption, $x \cdot \xi=|x| \xi_{\eta}$ and then, following from the formulas (5.1), (5.2) and (5.3), we have

$$
x \cdot \xi=|x|\left\{\xi_{n}(p)-\frac{1}{\tilde{S}_{n}(p)} \sum_{j=1}^{n-1} \tilde{s}_{j}(p) \xi_{j}(s, t)+\sum_{j=1}^{k} \lambda_{j}(t) s_{j}^{2}\right\} .
$$

Hence the integral in the left-hand side of (5.4) can be written as follows:

$$
\int_{N(\tilde{S})} e^{i x \cdot \xi} \mu(\xi) \phi(\xi) d S(\xi)=\exp \left\{i|x| \xi_{n}(p)\right\} \int_{|t| \leq \epsilon} \Psi(|x|, t) d t,
$$

where

$$
\Psi(|x|, t)=\int_{s} \exp \left\{i|x| \sum_{1}^{k} \lambda_{j}(t) s_{j}^{2}\right\} \psi(s, t) d s
$$

with

$$
\psi(s, t)=\left.\mu(\xi) \phi(\xi) J(\xi, \eta)\right|_{\xi=(s, t)} \exp \left[\frac{-i|x|}{\tilde{S}_{n}(p)} \sum_{1}^{n-1} \tilde{s}_{j}(p) \xi_{j}(s, t)\right] .
$$

For each fixed $t$, by the arguments in W. Littman [19] and [20] for the case of nonzero Gaussian curvature, when $|x| \rightarrow \infty$,

$$
\Psi(|x|, t)=\left(\frac{\pi}{2}|x|^{-1}\right)^{k / 2}(1+i)^{d^{+}}(1-i)^{d^{-}} \frac{\psi(0, t)}{\left|K_{k}(0, t)\right|^{1 / 2}}+O\left(|x|^{-(k+1) / 2}\right)
$$

Therefore we have the assertion of the theorem.

For the various directions $\omega$ of $x=|x| \omega$ near $\Re(p) \in \Omega_{k}$, we have the following approximations at infinity with support near $p$. 
Corollary 5.1. For $\omega$ in the direction of $\Re(p)$,

$$
\{\mu \phi\}^{\wedge}(|x| \omega)=c(p)|x|^{-k / 2} e^{i|x| \omega \cdot p}+O\left(|x|^{-(k+1) / 2}\right) \text { when }|x| \rightarrow \infty .
$$

Indeed, $\omega$ in the direction of $\Re(p)$ yields $\tilde{S}_{j}(p)=0, j=1, \cdots, n-1$, in the left-hand side of the formula (5.4). Hence the integrand is independent of $|x|$ and then we have the assertion by (5.5) implying

$$
c(p) \equiv\left(\frac{\pi}{2}\right)^{k / 2}(1+i)^{d^{+}}(1-i)^{d^{-}} \cdot \int_{|t| \leq \epsilon} \mu_{1}(t) d t .
$$

Corollary 5.2. For $\omega$ far away from the direction of $\Re(p)$ but not orthogonal to $\Re(p)$,

$$
\{\mu \phi\}^{\wedge}(|x| \omega)=O\left(|x|^{-d}\right) \text { when }|x| \rightarrow \infty
$$

for any number $d>0$.

Indeed, since $\omega$ is not orthogonal to $\Re(p), \tilde{S}_{n}(p) \neq 0$ in $(5.4)$ and, since $\omega$ is not in the direction of $\Re(p)$, there is a $j, j=1, \cdots, n-1, \tilde{s}_{j}(p) \neq 0$. The integration by parts in the integral of the right-hand side of (5.4) gives the assertion.

Theorem 5.1. For a $S \in \mathbb{S}_{k}$ and density function $\mu$ with compact support on $N(\tilde{S})$ and $\Omega_{k} \neq \varnothing$,

$$
\mu^{\wedge}(x)=O\left(|x|^{-k / 2}\right) \text { when } x \rightarrow \infty,
$$

uniformly in the direction.

Proof. For the result in (5.10) consider first that the unit vector $\omega$ is orthogonal to $\Omega_{k}$. We claim that, for some $b>(k+1) / 2$,

$$
\mu^{\wedge}(|x| \omega)=O\left(|x|^{-b}\right) \text { when }|x| \rightarrow \infty .
$$

Indeed, for convenience we assume $\omega=(0, \cdots, 0,1)$. If $\omega \in \Omega_{d}$ for some $d>k$, for each $p \in N_{d}$ with $\Re(p)=\omega$, Corollaries 5.1 and 5.2 imply that $[\mu \phi]^{\wedge}(|x| \omega)$ satisfies (5.1) where supp $\phi$ is contained in a small neighborhood of $p$. Next if $\omega$ is orthogonal to $\Omega_{d}$ for all $d=k, \cdots, n-1$, it is impossible that $\tilde{S}_{j}(\xi)=0$ for all $j \neq n$ (and then $\widetilde{S}_{n}(\xi) \neq 0$ by condition (ii)), since $\omega=(0, \ldots, 0,1)$ is perpendicular to all $\mathfrak{N}(\xi)=\operatorname{grad} \tilde{S}(\xi) / \operatorname{grad} \tilde{S}(\xi) \mid$. Hence there is $j, 1 \leq j<n-1$, say $j=1$ for $p \in N(P)$, such that $\tilde{S}_{1}(p) \neq 0$. Choosing $\phi \in C_{0}^{\infty}\left(R^{n}\right)$ with support near $p$ on which $\left|\tilde{S}_{1}(\xi)\right|>1 / 2$, we have, by Theorem 1.1 ,

$$
\begin{aligned}
{[\mu \phi]^{\wedge}(|x| \dot{\omega}) } & =\int_{N(\tilde{S}) \cap J} e^{i x \cdot \xi}[\mu \phi](\xi) d S(\xi) \\
& =\int_{\xi^{\prime}} \exp \left\{i|x| \xi_{n}\right\} \frac{\mu \phi}{\tilde{S}_{1}}\left(\xi_{1}\left(0, \xi^{\prime}\right), \xi^{\prime}\right) d \xi^{\prime}=O\left(|x|^{-\nu}\right) \text { when }|x| \rightarrow \infty
\end{aligned}
$$


for any $\nu>0$. Hence the compactness of supp $\mu$ and the partition of unity yield the claim of (5.10).

From this and Corollary 5.2, it suffices to consider the direction $\omega \in \Omega_{k}$. For a point $p \in N_{k}$ with $\Re(p)=\omega$, Lemma 5.1 implies the existence of $\phi$ with support in a small neighborhood of $p$ such that (5.7) holds. By $\overline{N_{k}}$ compact, there is a finite number of points $p_{j}$ and functions $\phi_{j}$ which form the partition of unity with respect to $\overline{N_{k}}$ and satisfy (5.7) with $\phi=\phi_{j}, d^{ \pm}=d^{ \pm}\left(p_{j}\right), p=p_{j}$, and $c(p)=c\left(p_{j}\right)$. Let $\phi_{0} \in C_{0}^{\infty}\left(R^{n}\right), 0 \leq \phi_{0} \leq 1$ and $\left\{\phi_{0}, \phi_{j}\right\}$ form a partition of unity corresponding to supp $\mu$. Since $\Re(\xi), \xi \in$ supp $\phi_{0}$, is far away from $\omega,\left\{\mu \phi_{0}\right\}^{\wedge}$ satisfies (5.8) with $\Re(\xi)$ either perpendicular, or not, to $\omega$, by the last claim or Corollary 5.2. Therefore we have, when $|x| \rightarrow \infty$,

$$
\mu^{\hat{\imath}}(|x| \omega)=\left(\frac{\pi}{2}|x|^{-1}\right)^{k / 2} \sum_{j} c\left(p_{j}\right) \exp \left\{i|x| \omega \cdot p_{j}\right\}+O\left(|x|^{-(k+1) / 2}\right) .
$$

This ends the proof of the theorem.

\section{BIBLIOGRAPHY}

1. A. S. Besicovitch, Almost periodic functions, Cambridge Univ. Press, New York, 1932.

2. Salomon Bochner and W. T. Martin, Several complex variables, Princeton Math.

Series, vol. 10, Princeton Univ. Press, Princeton, N. J., 1948. MR 10, 366.

3. Kuang-Ho Chen, Symmetrization of distributions and its application, Trans. Amer. Math. Soc. 162 (1971), 455-471.

4. Leon Ehrenpreis, Solution of some problems of division. I. Divisions by a polynomial of derivation, Amer. J . Math. 76 (1954), 883-903. MR 16, 834.

5. - Solution of some problems of division. II. Division by a polynomial of derivation, Amer. J. Math. 77 (1955), 286-292. MR 16, 1123.

6. - Solutions of some problems of division. III. Division in the spaces $\mathcal{D}^{\prime}, K$, $2_{A}$, O, Amer. J. Math. 78 (1956), 685-715. MR 18, 746.

7. - Solutions of some problems of division. IV. Invertible and elliptic operators, Amer. J. Math. 82 (1960), 522-588. MR 22 \#9848.

8. - Solutions of some problems of division. V. Hyperbolic operators, Amer. J. Math. 84 (1962), 324-348. MR 26 \#480.

9. Avner Friedman, Generalized functions and partial differential equations, PrenticeHall, Englewood Cliffs, N. J., 1963. MR 29 \#2672.

10. Entire solutions of partial differential equations with constant coefficients, Duke Math. J. 31 (1964), 235-240. MR 28 \#5255.

11. I. M. Gel'fand and G. E. Šilov, Generalized functions. Vol. 1: Operations on them, Fizmatgiz, Moscow, 1958; English transl., Academic Press, New York, 1964. MR 20 \#4182; MR $29 \# 3869$.

12. - Generalized functions. Vol. 2: Spaces of fundamental functions, Fizmatgiz, Moscow, 1958; English transl., Academic Press; Gordon and Breach, New York, 1968. MR $21 \# 5142 \mathrm{a} ; \quad$ MR 37 \#5693.

13. - Generalized functions. Vol. 3: Some questions in the theory of differential equations, Fizmatgiz, Moscow, 1958; English transl., Academic Press, New York, 1967. MR 21 \#5142b; MR 36 \#506.

14. V. V. Grušin, On Sommerfeld-type conditions for a certain class of partial differential equations, Mat. Sb. 61 (103) (1963), 147-174; English transl., Amer. Math. Soc. Transl. (2) 51 (1966), 82-112. MR $28 \# 346$. 
15. Lars Hörmander, Estimates for translation invariant operators in $L^{p}$ spaces, Acta Math. 104 (1960), 93-140. MR 22 \#12389.

16. - Hypoelliptic convolution equations, Math. Scand. 9 (1961), 178-184. MR $25 \# 3265$.

17. On the range of convolution operators, Ann. of Math. (2) 76 (1962), 148-170. MR $25 \# 5379$.

18. Linear partial differential operators, Die Grundlehren der math. Wissenschaften, Band 116, Academic Press, New York; Springer-Verlag, Berlin, 1963. MR 28 \#4221.

19. Walter Littman, Fourier transforms of surface-carried measures and differentiability of surface averages, Bull. Amer. Math. Soc. 69 (1963), 766-770. MR 27 \#5086.

20. Decay at infinity of solutions to partial differential equations with constant coefficients, Trans. Amer. Math. Soc. 123 (1966), 449-459. MR 33 \#6110.

21. Maximal rates of decay of solutions of partial differential equations, Arch. Rational Mech. Anal. 37 (1970), 11-20. MR 41 \#2197.

22. B. Malgrange, Existence et approximation des solutions des équations aux dérivées partielles et des équations de convolution, Thesis, Paris, 1955; Ann. Inst. Fourier (Grenoble) $6(1955 / 56), 271-355$. MR 19, 280.

23. L. Schwartz, Théorie des distributions. Tome I, Actualités Sci. Indust., no. 1091, Hermann, Paris, 1950. MR 12, 31.

24. - Théorie des distributions. Tome II, Actualités Sci. Indust., no. 1022, Hermann, Paris, 1951. MR 12, 833.

25. J. F. Tréves, Lectures on linear partial differential equations with constant coefficients, Notas de Matemática, no. 27, Instituto de Matemática Pura e Aplicada do Conselho Nacional de Pesquisas, Rio de Janeiro, 1961. MR 27 \#5020.

26. - Linear partial differential equations with constant coefficients: Existence, approximation and regularity of solutions, Math. and its Appl., vol. 6, Gordon and Breach, New York, 1966. MR 37 \#557.

27. - Differential polynomials and decay at infinity, Bull. Amer. Math. Soc. 66 (1960), 184-186. MR 22 \#8227.

28. M. I. Višik and G. I. Eskin, Convolution equations in a bounded region, Uspehi Mat. Nauk 20 (1965), no. 3 (123), 89-152 = Russian Math. Surveys 20 (1965), no. 3, 85-151. MR 32 \#2741.

29. - Convolution equations in a bounded region in spaces with weighted norms, Mat. Sb. 69 (111) (1966), 65-110; English transl., Amer. Math. Soc. Transl. (2) 67 (1968), 33-82. MR $36 \# 1935$.

30. Z. Zielé́ny, Hypoelliptic and entire elliptic convolution equations in subspaces of the space of distributions. I, Studia Math. 28 (1966/67), 317-332. MR 36 \#5528.

31. Hypoelliptic and entire elliptic convolution equations in subspaces of the space of distributions. II, Studia Math. 32 (1969), 47-59. MR 40 \#1773.

DEPARTMENT OF MATHEMATICS, LOUISIANA STATE UNIVERSITY, NEW ORLEANS, LOUISIANA 70122 